\title{
Exploring the Role of Self-Kindness in Making Healthier Eating Choices: A Preliminary Study
}

\author{
Misba Hussain $^{1}$ (D) Helen Egan ${ }^{1} \cdot$ Rebecca Keyte $^{1} \cdot$ Michail Mantzios $^{1}$ \\ Accepted: 28 October 2020 / Published online: 23 November 2020 \\ (C) International Society of Behavioral Medicine 2020
}

\begin{abstract}
Background Food selection is an important factor in the prevalence of obesity, and regularly choosing nutrient-poor high-energy foods can have negative health consequences. The present study tested whether the use of mindful construal diaries (MCD) combined with self-kindness could promote healthier eating choices.

Method Ninety participants were randomly assigned to either a self-kindness to the mind condition or self-kindness to the mind and body condition, and were served M\&Ms and grapes as an unhealthy and healthy option, respectively.

Results The results suggested that a difference in consumption was found between the two conditions, with participants in the self-kindness to the mind condition consuming significantly more grapes than those in the self-kindness to the mind and body condition. However, this difference was restricted to those who are usually low consumers of fruit and vegetable.

Conclusion The effect of self-kindness towards promoting heathier eating whilst considering the body thus appears to require further investigation. Limitations and recommendations for future research are discussed.
\end{abstract}

Keywords Self-compassion $\cdot$ Self-kindness $\cdot$ Food choices $\cdot$ Healthy eating $\cdot$ Unhealthy eating

\section{Introduction}

Encouraging healthier food choices appears to be crucial in improving weight management and addressing the current weight-related chronic conditions, such as type 2 diabetes, cardiovascular disease and COVID-19 [1, 2]. Mindfulnessbased interventions have shown to positively influence healthier eating behaviours, and the inclusion of self-compassion to reinforce one's ability to be less self-critical is a foundational element within mindfulness practices [3, 4]. Recent literature has indicated that self-kindness, an element within self-compassion, may pose as a problematic area in health behaviour research and healthy changes within eating $[5,6]$. The present research attempted to identify whether being kind to oneself is helpful (or not) when exploring a mindful eating intervention targeting decision making and consumption [7].

Misba Hussain

Misba.hussain@mail.bcu.ac.uk

1 Department of Psychology, Faculty of Business, Law and Social Sciences, Birmingham City University, The Curzon Building, 4 Cardigan St., Birmingham B4 7BD, UK
Mindfulness has been described as purposely paying attention to present moment experiences, non-judgementally $[8,9]$. Mindful eating, a term that describes the adaptation of mindfulness onto the behaviour of eating, has shown significant changes in weight regulation $[7,10,11]$. Eating is often described as an automatic and over-learned behaviour [12]. For example, people may eat simply in the presence of food, and not necessarily because they are hungry [13]. It has been suggested that engaging in a mindful eating approach encourages one to place a greater focus upon their internal cues of hunger and satiety, reducing the influence of external cues and eventually leading to healthier eating behaviours $[10,14]$. In an attempt to induce a state of mindful eating, a "mindful concrete construal" diary (MCD) was developed [7]. The MCD combines elements of mindfulness and self-compassion, and using the MCD has demonstrated improvements in participants' mindfulness levels and eating patterns, such as reduced caloric intake [7, 15].

Self-compassion is described as treating oneself with the same understanding and concern as one would treat loved ones with when experiencing difficulties [16]. It consists of three main elements: self-kindness, common humanity and mindfulness [16]. Literature suggests that the combination of both mindfulness and self-compassion promotes positive 
outcomes in eating behaviours, well-being and weight management $[17,18]$. Yet, it is uncertain as to which specific elements of self-compassion improve healthier choices around food. One area that appears to be problematic within the concept of self-compassion is self-kindness [5]. Whilst it is commonly believed that being kind to oneself is beneficial when trying to lose weight, the influence that self-kindness may have upon physiological health is not so straightforward [5, 6]. For example, recent research found wide separations in behaviours regarding self-kindness; for some people, selfkindness involved binge drinking or over-indulging on their favourite foods, for others it consisted of taking a warm bath or eating a healthy meal [6]. Whilst the former group's behaviour may lead to negative health consequences, and only refers to perceived kindness to the mind, the latter group's behaviour relates to self-kindness of both the mind and body, and therefore is perhaps a truer model of self-compassion and the golden standard of self-care [16].

Whilst self-kindness may be a positive method of alleviating psychological distress, being kind to oneself does not necessarily mean that both physical and psychological health are attained simultaneously [6]. In practice, psychological health is often prioritised over physiological health, for example, someone who is on a weight loss diet and has had a stressful day may want to treat themselves by eating a chocolate cake instead of a portion of fruit because "they deserve it" [19]. By doing so, they are only showing perceived kindness to their mind, and if such behaviour is sustained continuously, it can make adhering to healthy eating difficult. Literature has also found a positive association between self-compassion and intake of fruit and vegetable [20], and more recently it was suggested that those who do not participate in the holistic care of self-compassion (i.e. displaying kindness to both the mind and body) may be less likely to consume nutrient-dense low-energy foods (e.g. fruit and vegetable) [6]. Priming participants who are usually low consumers of fruit and vegetable to be kind to both their mind and body may lead to a greater intake of healthier food choices than those who are high consumers of fruit and vegetable. This is because low consumers may be prompted to change their food choices in order to demonstrate kindness to their mind and body, whereas high consumers already appear to express self-compassion and self-kindness to their mind and body $[6,20]$. Therefore, exploring the state of self-kindness, and the affect that it may have upon eating behaviours, is an avenue that merits investigation.

This study will explore whether the MCD combined with self-kindness could promote healthier food choices. It is hypothesised that participants who simply consider the answers to the MCD questions combined with self-kindness to both the mind and body will be more likely to choose healthier food options than participants who consider the answers to the MCD combined with self-kindness solely to the mind. However, it is predicted that this effect will only occur in participants who are usually low consumers of fruit and vegetable, as they would be less likely to practise the holistic act of self-kindness on a daily basis [6].

\section{Method}

\section{Participants}

Ninety participants were recruited via opportunity sampling from a university in West Midlands, UK. Participants included both university and high school students, as well as parents and siblings of students. Given the low and high consumer subset of the population pool, an aim of exceeding a large effect size at power equalling 0.80 and $p=0.05$, participant numbers were set at 18 for each group, and 45 for a medium effect size. The current study achieved a large-medium effect size [21]. The average BMI of the sample was $M=24.32(S D=9.13)$ and age was $M=24.54(S D=10.83)$. Participants' self-identified ethnicities were White or White British $(n=55)$, Black African or Caribbean $(n=12)$, Asian $(n=19)$, Chinese $(n=$ $2)$, Arab $(n=1)$, and not specified $(n=1)$.

Eligibility Participants were informed via an information sheet and consent form that they were not eligible to participate if they had been diagnosed with an eating disorder or if they had any nut allergies. There were no other restrictions on participating.

\section{Experimental Conditions}

Participants were randomly assigned to one of the two experimental conditions. Participants in the self-kindness to the mind condition $(n=45)$ received a MCD [7] that was adapted with questions regarding kindness to one's mind and thoughts, such as "How do you feel and what passes through your mind now that you are eating this snack" and "How important is this snack for taking care of your thoughts and feelings right now." Participants in the self-kindness to the mind and body condition $(n=45)$ were presented with a MCD adapted with questions regarding kindness to both the mind and body. Sample items of the diary include "How could this snack be better at taking care of your thoughts and physiological health right now?" and "How are you taking care of your emotions and your physical health now that you are eating this snack?" Participants in both the conditions were asked to simply consider the answers to the adapted versions of the MCD in order to avoid any distractions that may occur from writing out answers [22, 23].

\section{Procedure}

The study was advertised as an experiment regarding food choices and personality in order to disguise the true aims of 
the study [15]. Experimental sessions took place between $12 \mathrm{pm}$ and $4 \mathrm{pm}$ and lasted approximately $25 \mathrm{~min}$. Upon arrival, participants received an information sheet, and those who provided informed consent were seated in individual cubicles. Participants' height and weight were first measured using a stadiometer and a digital scale, and they were then asked to complete demographic questions and a state self-compassion scale. Next, depending on the condition, participants were asked to either read the kindness to the mind MCD or kindness to the mind and body MCD for 2 min prior to receiving the M\&Ms and grapes [15]. Then, participants were provided with the portion of M\&Ms and grapes, and asked to continue reading and engaging with the MCD whilst eating. Participants were told they could choose any option of food they want (or both) and eat as much or as little as they prefer. After $5 \mathrm{~min}$, the experimenter asked participants to finish eating and they were asked to complete another state self-compassion scale and a cognitive restraint scale. Once participants had completed the questionnaires, they were debriefed about the study's true purpose, and thanked for their participation.

\section{Self-Report Measurements}

Demographics To assess background information, participants were asked questions on their age, ethnicity and how many hours prior they had eaten.

Hunger To assess hunger, participants were asked "How hungry do you feel right now" with responses ranging from 1 (not at all) to 5 (extremely hungry).

Fruit and vegetable consumption To assess daily fruit and vegetable consumption, participants were asked "How many portions of fruit and vegetable do you normally eat a day?" [24].

State self-compassion scale To assess state self-compassion, participants completed the state self-compassion scale pre and post experimental task [25], and the present study produced alpha of pre $(\alpha=0.84)$ and post $(\alpha=0.87)$.

Cognitive restraint scale To assess diet tendencies, participants completed the cognitive restraint subscale of the Three-Factor Eating Questionnaire [26], and the present study produced an alpha of $(\alpha=0.78)$. Participants completed all questionnaires, and there was no missing data.

\section{Food}

Participants in both conditions were provided with a $100-\mathrm{g}$ portion of M\&M peanuts (approximately 511 calories, $19 \mathrm{~g}$ fat, $72 \mathrm{~g}$ carbohydrate, $4 \mathrm{~g}$ protein) and a $100-\mathrm{g}$ portion of green and red grapes (approximately 67 calories, $0.2 \mathrm{~g}$ fat, $18 \mathrm{~g}$ carbohydrate, $0.7 \mathrm{~g}$ protein). The M\&Ms and grapes were served in two separate white bowls with the size amounting to width $15 \mathrm{~cm} \times$ length $15 \mathrm{~cm} \times$ height $8 \mathrm{~cm}$, and all food was sourced from UK Tesco stores.

\section{Data Analysis}

Shapiro-Wilk test was used to determine normality. A median split on the usual fruit and vegetable intake measure resulted in low consumers categorised as participants consuming 2 or less portions of fruit and vegetables a day, and high consumers labelled as participants consuming 2.5 or more portions of fruits and vegetables a day. $2 \times 2$ ANOVAs with state selfcompassion and energy intake from M\&Ms and grapes as dependent variables were conducted, and any significant interactions were followed up with $t$ tests. Participants who did not eat anything $(n=5)$ were excluded in a secondary analysis, and findings reported for the whole sample remained the same in regard to significance reporting. As such, all participants $(n=90)$ are included in the following analyses.

\section{Results}

\section{Participant Characteristics}

Fifty participants were labelled as low consumers of fruit and vegetables (daily portion: $M=1.51, S D=0.63$ ), whilst the remaining forty participants were identified as high consumers (daily portion: $M=3.98, S D=1.30$ ). Chi-square analysis revealed that gender was equal across the two conditions $\left(X^{2}(2)=0.2 .19, p=0.34\right)$, and $t$ tests found no significant differences in participants' hunger, prior hours eaten, BMI, age, fruit and vegetable consumption and cognitive restraint between the two conditions: all $p>0.12$. Inclusion of hunger, prior hours eaten, BMI, age, cognitive restraint and gender as covariates in the analyses did not affect the observed results for any of the dependent measures, and are therefore not discussed further.

\section{State Self-Compassion}

A $2 \times 2$ ANOVA indicated a significant main effect of time $\left(F_{(1,88)}=137.50, p<0.001, \eta_{\mathrm{p}}{ }^{2}=0.61\right)$ with state selfcompassion scores being significantly higher within the post condition $(M=78.72, S D=15.32)$ than the pre condition $(M=66.19, S D=12.85)$. There was no main effect of conditions $\left(F_{(1,88)}=0.80, p=0.38, \eta_{\mathrm{p}}{ }^{2}=0.01\right)$, and there was no significant interaction between time $\times$ condition $\left(F_{(1,88)}=\right.$ $0.004, p=0.95, \eta_{\mathrm{p}}{ }^{2}=0.001$ ).

\section{Food Selection: Overall Intake}

A $2 \times 2$ ANOVA indicated no significant interaction between condition $\times$ usual fruit and vegetable intake $\left(F_{(1,86)}=0.01\right.$, 
$\left.p=0.95, \eta_{\mathrm{p}}{ }^{2}=0.001\right)$, no significant main effect of conditions $\left(F_{(1,86)}=1.07, p=0.31, \eta_{\mathrm{p}}{ }^{2}=0.01\right)$ nor of usual fruit and vegetable intake $\left(F_{(1,86)}=2.43, p=0.12, \eta_{\mathrm{p}}{ }^{2}=0.03\right)$ on participants' total calorie intake (see Table 1).

\section{Food Selection: M\&Ms}

A $2 \times 2$ ANOVA indicated no significant interaction between condition $\times$ usual fruit and vegetable intake $\left(F_{(1,86)}=0.11\right.$, $\left.p=0.75, \eta_{\mathrm{p}}{ }^{2}=0.001\right)$, no significant main effect of conditions $\left(F_{(1,86)}=0.95, p=0.33, \eta_{\mathrm{p}}{ }^{2}=0.01\right)$ nor of usual fruit and vegetable intake $\left(F_{(1,86)}=2.12, p=0.15, \eta_{\mathrm{p}}{ }^{2}=0.02\right)$ on participants' calorie intake of M\&Ms (see Table 1).

\section{Food Selection: Grapes}

A $2 \times 2$ ANOVA indicated a significant interaction between condition $\times$ usual fruit and vegetable interaction $\left(F_{(1,86)}=\right.$ 7.68, $\left.p=0.01, \eta_{\mathrm{p}}{ }^{2}=0.08\right)$ on participants' calorie intake of grapes. There was no significant main effect of conditions $\left(F_{(1,86)}=0.85, p=0.36, \eta_{\mathrm{p}}{ }^{2}=0.01\right)$, nor usual fruit and vegetable intake $\left(F_{(1,86)}=2.22, p=0.14, \eta_{\mathrm{p}}{ }^{2}=0.03\right)($ see Table 1$)$.

\section{Low Consumers: Grape Selection}

For low consumers, there was a significant effect of selfkindness on participants' calorie intake of grapes $(t(43.33)=$ 2.75, $p=0.01, d=0.77$ ), whereby participants in the selfkindness to the mind condition consumed significantly more grapes than participants in the self-kindness to the mind and body condition (see Table 1).

\section{High Consumers: Grape Selection}

For high consumers, there was no significant difference between the self-kindness to the mind and self-kindness to the mind and body conditions in participants' calorie intake of grapes $(t(38)=1.26, p=0.21, d=0.41)($ see Table 1$)$.

\section{Discussion}

The present study investigated whether or not the MCD combined with self-kindness could promote healthier food choices. Whilst a difference was found in the intake of healthy and unhealthy foods between the two conditions, it was limited and not consistent with the aforementioned hypotheses. The data suggested there were no significant differences in the consumption of M\&Ms or grapes between self-kindness to the mind condition and self-kindness to the mind and body condition. However, participants who were typically low consumers of fruit and vegetable consumed significantly more grapes in the self-kindness to the mind condition compared to those in the self-kindness to the mind and body condition. Therefore, the findings suggest that the concept of selfkindness may not be so apparent when promoting healthier food choices, especially amongst low consumers of fruit and vegetable.

One possible interpretation of the results is that low consumers of fruit and vegetable may have altered their behaviour and consumed more grapes in the self-kindness to the mind condition because of the effect of mindful eating. For example, literature on mindful eating has demonstrated positive outcomes on promoting healthier eating choices [10, 11], and although the questions within the MCD were priming participants to be kinder to their mind, the diary was also encouraging participants to be more mindful. Previous research that has investigated the original MCD involving mindfulness and self-compassion [7] found the diary to be significantly effective in promoting healthier eating choices [15]. Furthermore, priming participants who are usually low consumers of fruit and vegetable to be kind to their mind and body was predicted to promote a greater intake of healthier foods (i.e. grapes). However, the unexpected finding of low consumers displaying a greater intake of healthier foods in the self-kindness to the mind condition than those in the selfkindness to the mind and body condition appears to support the narrative that mindfulness may have been more influential
Table 1 Means and standard deviations of calories consumed

\begin{tabular}{lll}
\hline & Mind condition $(n=45)$ & Mind and body condition $(n=45)$ \\
\hline Total intake & $130.04(146.62)$ & $100.29(142.94)$ \\
M\&Ms & $105.24(137.88)$ & $80.21(132.64)$ \\
Grapes & $24.80(20.11)$ & $20.08(19.86)$ \\
Low consumers of fruit and vegetable $(n=50)$ & Mind condition $(n=26)$ & Mind and body condition $(n=24)$ \\
Grapes & $27.00(23.02)$ & $12.00(15.06)$ \\
M\&Ms & $83.69(118.00)$ & $65.07(113.48)$ \\
High consumers of fruit and vegetable $(n=40)$ & Mind condition $(n=19)$ & Mind and body condition $(n=21)$ \\
Grapes & $21.79(15.32)$ & $29.31(20.93)$ \\
M\&Ms & $134.74(159.84)$ & $97.52(152.66)$ \\
\hline
\end{tabular}

Standard deviations in parentheses 
than the concept of self-kindness. Literature solely exploring mindful eating practices demonstrates a clear narrative of promoting healthier eating behaviours and weight regulation [7, 10]. Although the improvements in self-compassion scores post intervention within the current study suggest that selfkindness was promoted, the concept of self-kindness within eating behaviours is still a novel and rather complex concept $[5,6]$. Therefore, future research should use self-kindness as a sole intervention without the combination of mindfulness in order to thoroughly explore its effect on eating behaviours.

Moreover, the process of thinking about being kind to oneself may have induced more self-consciousness, and in effect promoted healthier eating behaviours [4]. For example, commercials on lowering sugar intake highlight the negative associations of sugar on the body. As a result, people may consciously adapt their food choices in order to promote their physiological health [27]. The notion of promoting physical health may thus require a greater focus on mind elements, which could in effect result in better decision making around food.

\section{Limitations and Future Directions}

There are some potential limitations to the present study that require further discussion and investigation. First, the present study did not include a control group that was exposed to a non-self-kindness condition, and therefore did not allow an assessment on whether self-kindness had any significant direct effect. Future studies should include such a condition to allow a direct assessment on the effect of self-kindness in comparison to a conventional version of the intervention. Additionally, considering how some analyses were underpowered when considering a medium effect size to be optimal in this type of research, future research needs to account for more participants.

Furthermore, whilst previous studies have used a similar time frame as the current research [28], and the current findings do suggest that hunger or hours prior eaten had no effect on the intake of healthy versus unhealthy foods. It may be possible that the time participants ate during the study affected their consumption [29]. Additionally, although participants with eating disorders and nut allergies were excluded from the current study, it could be possible that participants who had illnesses, such as type 2 diabetes, may have been prohibited from consuming high-sugar snacks. Therefore, controlling for such factors within future research may serve an enhanced and more tightly controlled experiment.

Finally, there was little variability in the BMI of the sample used, and people who are overweight or obese may have responded differently. For example, overweight and obese people may more often choose psychological health over physiological [30], and including these populations may provide different results.

\section{Conclusion}

The findings from the preliminary study suggest that mindfulness combined with self-kindness to the mind can encourage healthier eating, but this effect is limited, and restricted to lower consumers of fruit and vegetable. The concept of selfkindness requires further research to understand further its impact on eating behaviours and weight regulation.

Authors' Contributions MH designed the study, collected data, conducted data analyses and wrote the manuscript. HE, RK and MM supported the study and critically reviewed the manuscript. All authors gave their final approval of the manuscript.

\section{Compliance with Ethical Standards}

Conflict of Interest The authors declare that they have no conflict of interest.

Ethical Approval All procedures have been approved by the institutional research committee of the University and have been performed in accordance with the ethical standards laid down in the 1964 Declaration of Helsinki and its later amendments.

Informed Consent Informed consent was obtained from all individual participants included in this study.

\section{References}

1. Manna P, Jain SK. Obesity, oxidative stress, adipose tissue dysfunction, and the associated health risks: causes and therapeutic strategies. Metab Syndr Relat Disord. 2015;13(10):423-44.

2. Kassir R. Risk of COVID-19 for patients with obesity. Obes Rev. 2020;21(6):e13034.

3. Braun T, Park C, Conboy LA. Psychological well-being, health behaviors, and weight loss among participants in a residential, Kripalu yoga-based weight loss program. Int J Yoga Ther. 2012;22(1):9-22.

4. Mantzios M, Wilson JC. Exploring mindfulness and mindfulness with self-compassion-centered interventions to assist weight loss: theoretical considerations and preliminary results of a randomized pilot study. Mindfulness. 2015;6(4):824-35.

5. Mantzios M, Egan H. On the role of self-compassion and selfkindness in weight regulation and health behavior change. Front Psychol. 2017;8:229.

6. Egan H, Mantzios M. A qualitative exploration of self-kindness and "treating oneself" in contexts of eating, weight regulation and other health behaviors: implications for mindfulness-based eating programs. Front Psychol. 2018;9:880.

7. Mantzios M, Wilson J. How concrete construals can become mindful: a novel approach of developing mindfulness and selfcompassion to assist weight loss. Psychol Health. 2014;29(4):1-21.

8. Bishop S, Lau M, Shapiro S, Carlson L, Anderson N, Carmody J, et al. Mindfulness: a proposed operational definition. Clin Psychol Sci Pract. 2006;11(3):230-41.

9. Kabat-Zinn J. Full catastrophe living: using the wisdom of your body and mind to face stress, pain and illness. New York: Delacourt; 1990.

10. Dalen J, Smith B, Shelley B, Sloan A, Leahigh L, Begay D. Pilot study: mindful eating and living (MEAL): weight, eating behavior, 
and psychological outcomes associated with a mindfulness-based intervention for people with obesity. Complement Ther Med. 2010;18(6):260-4.

11. Mantzios M, Giannou K. Group vs. single mindfulness meditation: exploring avoidance, impulsivity, and weight management in two separate mindfulness meditation settings. Appl Psychol: Health Well-Being. 2014;6(2):173-91.

12. Cohen D, Farley TA. Peer reviewed: eating as an automatic behavior. Prev Chronic Dis. 2008;5(1).

13. Painter J, Wansink B. How visibility and convenience influence candy consumption. Appetite. 2002;38(3):237-8.

14. Mantzios M, Egan H, Hussain M, Keyte R, Bahia H. Mindfulness, self-compassion, and mindful eating in relation to fat and sugar consumption: an exploratory investigation. Eat Weight DisordStud Anorexia, Bulimia Obes. 2018;23(6):833-40.

15. Hussain M, Egan H, Keyte R, Carter R, Mantzios M Mindful construal diaries: promoting healthier eating choices. Submitted

16. Neff $K$. The development and validation of a scale to measure selfcompassion. Self Identity. 2003;2(3):223-50.

17. Mantzios M, Wilson J, Linnell M, Morris P. The role of negative cognition, intolerance of uncertainty, mindfulness, and selfcompassion in weight regulation among male army recruits. Mindfulness. 2014;6(3):545-52.

18. Shapiro S, Astin J, Bishop S, Cordova M. Mindfulness-based stress reduction for health care professionals: results from a randomized trial. Int J Stress Manag. 2005;12(2):164-76.

19. Taylor C, Webb T, Sheeran P. 'I deserve a treat!': justifications for indulgence undermine the translation of intentions into action. Br J Soc Psychol. 2013;53(3):501-20.

20. Sirois FM, Kitner R, Hirsch JK. Self-compassion, affect, and health-promoting behaviors. Health Psychol. 2015;34(6):661-9.

21. Cohen J. Statistical power analysis for the behavioral sciences: Academic press; 2013.
22. Papies EK, Hamstra P. Goal priming and eating behavior: enhancing self-regulation by environmental cues. Health Psychol. 2010;29(4):384-8.

23. Foerde K, Knowlton BJ, Poldrack RA. Modulation of competing memory systems by distraction. Proc Natl Acad Sci. 2006;103(31): 11778-83.

24. Robinson E, Fleming A, Higgs S. Prompting healthier eating: testing the use of health and social norm based messages. Health Psychol. 2014;33(9):1057-64.

25. Breines JG, Chen S. Activating the inner caregiver: the role of support-giving schemas in state self-compassion. J Exp Soc Psychol. 2013;49(1):58-64.

26. Karlsson J, Persson LO, Sjöström L, Sullivan M. Psychometric properties and factor structure of the Three-Factor Eating Questionnaire (TFEQ) in obese men and women. Results from the Swedish Obese Subjects (SOS) study. Int J Obes. 2000;24(12):1715-25.

27. Elliott-Green A, Hyseni L, Lloyd-Williams F, Bromley H, Capewell S. Sugar-sweetened beverages coverage in the British media: an analysis of public health advocacy versus pro-industry messaging. BMJ Open. 2016;6(7):e011295.

28. Hussain M, Egan H, Keyte R, Mantzios M (2020) Exploring the effects of mindfulness and self-distancing on chocolate intake after a negative state affect. J Cogn Enhanc 1-10

29. Cross AT, Babicz D, Cushman LF. Snacking patterns among 1,800 adults and children. J Am Diet Assoc. 1994;94(12):1398-403.

30. Hendrikse JJ, Cachia RL, Kothe EJ, McPhie S, Skouteris H, Hayden MJ. Attentional biases for food cues in overweight and individuals with obesity: a systematic review of the literature. Obes Rev. 2015;16(5):424-32.

Publisher's Note Springer Nature remains neutral with regard to jurisdictional claims in published maps and institutional affiliations. 